\title{
Smokeless tobacco use among professional baseball players: survey results, 1998 to 2003
}

\author{
H H Severson, K Klein, E Lichtensein, N Kaufman, C T Orleans
}

Tobacco Control 2005;14:31-36. doi: 10.1136/tc.2004.007781

See end of article for authors' affiliations

.....................

Correspondence to: Herbert H Severson, Oregon Research Institute, 1715 Franklin Blvd, Eugene OR 97403, USA; herb@ori.org

Received 19 March 2004 Accepted 27 October 2004

\begin{abstract}
Objective: The use of smokeless tobacco (ST) (snuff and chewing tobacco) has long been associated with baseball in the USA. This article reviews six years of survey data from major and minor league baseball players to evaluate trends in tobacco use and quitting patterns over time in order to gain insight into the effects of past interventions and to document continued intervention needs.

Method: Surveys were distributed by athletic trainers to major and minor league professional baseball players during spring training session in the six years from 1998 to 2003 . The surveys were anonymous and identified only by team, level of league, and other self reported demographic data.

Results: ST use among professional baseball players remains much higher than among young males in the general population, and use is most prevalent among white non-Hispanic players. There was a significant decrease in ST use among minor league players from 1998 to 2003, with seven day self reported use declining from $31.7 \%$ in 1998 to $24.8 \%$ in 2003 . No significant year to year changes were observed for major league players. Major league players' self reported past week use rates, estimated at $35.9 \%$ in 1998 and at $36 \%$ in 2003, were consistently higher than those of minor league players. Self reported prevalence of past month cigarette and cigar smoking was much lower than ST use for both major and minor league players.

Conclusions: Six years of survey data confirm a continuing high use of ST among professional baseball players. Results suggest that the effects of the broad spectrum ST control efforts launched over the past decade may have been stronger among minor than major league players. Stronger policy interventions at the major league level and multi-level efforts, including programmes to increase the use of effective quitting aids and assistance, at both levels of play are needed. Future research is needed to further clarify changes in ST practices among professional players and set policy intervention directions.
\end{abstract}

$\mathrm{T}$ here has been ongoing concern about the prevalence of smokeless or "spit" tobacco (ST) use among professional baseball players. Use of chewing tobacco and snuff, historically part of US baseball culture, became much more prevalent in the late 1970s and early 1980s in response to aggressive marketing and promotion targeted toward professional baseball players. By the mid 1980s and early 1990s the prevalence of ST use among professional baseball players was about twice as high as that of the general population of males in the same age range. ${ }^{1-3}$ Therefore, baseball players provide a prime target for researchers to investigate the precursors, motivations, and health consequences of ST use and cessation.

Baseball players are particularly important to target for intervention because they are role models for young children, teens, and young adults in the USA, especially those who play baseball or other sports. The use of ST is tied to the culture of baseball, not just within the clubhouse but also in the public eye. From 1986 to 1998, ST had a visible presence in televised World Series games, ${ }^{4-6}$ providing free advertising for tobacco companies. From 1999 through 2001, no visible ST use was documented, but 5.1 minutes of obvious ST use was documented in the fourth game of the 2002 World Series (Jones R, personal communication, 24 October 2002). These decreases reflect changing major league baseball policy prohibiting onfield use by minor league players, as well as comprehensive decade long efforts to curb use among major league players.

Analysis of six surveys of ST use in professional baseball demonstrated that usage rates among major league players rose in the 1980s to a high of $46 \%$ in 1987, and then declined to $43 \%$ in 1990 and $31 \%$ in $1997 .{ }^{1-3} 7$ Use of ST among minor league players also showed a steady decline across those years, dropping remarkably from $68 \%$ of players reporting current use in 1987 to $29 \%$ in $1997 .{ }^{1}$
The association between baseball and ST is present in college and high school players as well. In a 1991 survey, college varsity baseball players were twice as likely to use ST as football players, with $52 \%$ of baseball players reporting regular use. ${ }^{8}$ Varsity baseball players were more than twice as likely to use ST as intramural baseball players. ${ }^{9}$ While rates of regular use of ST are lower among high school baseball players, occasional use of and experimentation with ST is quite prevalent. Studies of high school players have found current use rates ranging from 6-15\%, with 35-46\% having used ST at least once. ${ }^{10}$ The majority of professional baseball players began using ST in their late teens, with a median age of initiation between $17-18$ years. ${ }^{211} 12$

Although ST use is relatively high among professional baseball players, many players have quit. Among professional baseball players, between $12.6-16.5 \%$ report they are former ST users. ${ }^{211}$ Another study found that almost half of current users $(47 \%)$ had made at least one previous quit attempt. ${ }^{3}$ The primary reason players have reported for wanting to quit was "health concerns". Among college players, $4.5-11 \%$ had previously used ST regularly, but were no longer users, ${ }^{89}$ and $10 \%$ of high school baseball players reported having quit. ${ }^{13}$

While there are no restrictions on ST use by major league baseball players, there has been a ban on the use of ST by minor league players since $1992 .{ }^{13}$ The use of ST has also been banned by the National Collegiate Athletic Association (NCAA) for college baseball players since 1994. ${ }^{14}$ Similar bans also exist for youth baseball leagues such as Little

Abbreviations: NCAA, National Collegiate Athletic Association; NSTEP, National Spit Tobacco Education Program; PBATS, Professional Baseball Athletic Trainers Society; ST, smokeless tobacco 
League, Babe Ruth League, American Legion, and USA Olympic Baseball. ${ }^{15}$

In order to address the growing use of spit tobacco among young men, and especially among baseball players, Oral Health America's National Spit Tobacco Education Program (NSTEP) was founded in 1994 with the goal of educating major and minor league players and their trainers and fans about the harms of ST use and addiction, and about effective cessation methods. In 1998 NSTEP began offering soft tissue oral examinations, cessation counselling, and educational presentations on the causes and effects of oral cancer to major and minor league players and coaching staff during spring training. Additional intervention involved providing cessation training to athletic trainers, distribution of nontobacco oral substitutes, and public service announcements at the games and on televised broadcasts.

Although previous studies provide some data on ST use among professional baseball players over the past two decades, most were one time surveys that sampled only a small subset of professional teams. The surveys described in this paper were conducted to update findings from previous surveys and provide useful data on cumulative effects of broad spectrum policy changes and targeted interventions on professional baseball players. The results of this survey provide information to guide continued and future intervention efforts.

Cross sectional surveys of major and minor league players making up the spring training rosters of all professional clubs were conducted yearly at spring training from 1998 to 2003. Surveys were designed and conducted by a team of investigators at the Oregon Research Institute in collaboration with Professional Baseball Athletic Trainers Society (PBATS) trainers and under the auspices of NSTEP.

The aims of these annual surveys were to examine changes in patterns of ST use and cessation over time among both major and minor league players, and to gain insight into the cumulative impact of over a decade of multi-level intervention strategies designed to curb use and promote cessation within this population. Survey questions examined general ST use and cessation patterns, related tobacco use patterns, and exposure to varied elements of the NSTEP programmes. Included in this paper are the results from the six years (1998 to 2003) of this survey.

\section{METHODS}

\section{Recruitment}

Players were recruited from all 30 major league organisations during spring training from 1998 through 2003. During spring training both major and minor league players are housed in the same complex, which allowed us to send one packet of surveys to the head athletic trainer of each club for distribution to both major and minor league players. Minor league players at three levels (single A, double A, and triple A leagues) were included in those surveyed. All surveys were anonymous; only team identification was obtained. Therefore, longitudinal tracking of individual players' tobacco use over time was not possible.

The number of survey respondents ranged annually from 1356 to 2442 minor league players and 136 to 454 major league players (table 1). A full minor league spring training roster can include up to 200 players, as these players will subsequently be assigned to five or six minor league teams at different levels of minor league baseball. A major league team roster during spring training typically has 50-60 players trying to make the final roster. Approximate return rates (based on the conservative assumption that each team invites 250 players to spring training) were: $28 \%$ in 1998; $23 \%$ in $1999 ; 25 \%$ in $2000 ; 33 \%$ in $2001 ; 35 \%$ in 2002 ; and $31 \%$ in 2003. Return rates were affected by both athletic trainers' cooperation in distributing the surveys and players' choosing to complete and return them. Response rates for different teams varied widely each year, and varied from year to year within a particular team. Although we received surveys from 20-25 of the 30 teams each year, only about a quarter of the teams returned more than 100 surveys and approximately one third returned 50 or fewer surveys. Therefore while some teams were adequately represented in the annual sample, other teams were represented by only a small proportion of their players.

\section{RESULTS}

\section{Smokeless tobacco use}

Players' reported seven day point prevalence of ST use is shown in table $1 .{ }^{*}$ ST use among major league players surveyed showed no overall change in prevalence with $35.9 \%$ reported in 1998 and $36.0 \%$ reported in $2003\left(\chi^{2}(4\right.$, $\mathrm{n}=1508)=2.7, \mathrm{p}>0.05$ ) (major league players were not surveyed in 2001). Post hoc $\chi^{2}$ analyses comparing use rates from each year to the subsequent year found no significant yearly changes in use. Self reported ST use prevalence was consistently higher among major league than minor league survey respondents.

ST use among minor league survey respondents did show a significant overall change in usage rates over time, declining from $31.7 \%$ in 1998 to $24.8 \%$ in $2003\left(\chi^{2}(5, \mathrm{n}=\right.$ $11589)=29.0, \mathrm{p}<0.001)$. This was primarily driven by a substantial drop from 1998 to $1999\left(\chi^{2}(1, \mathrm{n}=3156)=10.8\right.$, $\mathrm{p}<0.001)$. Post hoc $\chi^{2}$ analyses found no other significant changes in ST use from year to year, with rates remaining fairly steady from 1999 through 2003, around 25-26\%.

The surveys also provided data on frequency of ST use among regular users (those who reported using at least once in the past seven days) (table 2). The percentages of players who used ST on a daily basis showed a slight drop over the years of the survey. Among players who were regular users, the average amount of ST consumed remained fairly constant. Those who reported regular use of ST reported a mean of five days' use per week, and that a tin of tobacco lasted them an average of about four and a half days.

Each year players were asked to report their race or ethnicity. In 1998 there was a very low response rate (51\%) to this question because of the placement and wording of the question. Therefore, race data are reported for 1999-2003 only. Table 3 displays the percentage of players within each racial or ethnic group who reported current ST use each year. Major league and minor league players reported very similar patterns of ST use by ethnicity; therefore, we collapsed across league and the numbers shown in the table represent all players. Given the low numbers of native Americans, Asians, and players checking "Other", meaningful comparisons could not be made, so we focused primarily on white, Latino, and African American players in making comparisons by race. White players reported the highest prevalence of ST use, approximately double that of Latino or African American players.

\section{Tobacco use and baseball}

In three of the six annual surveys (2000, 2001, 2003), respondents were asked how their ST use changed during the

*The data in table 1 are based on players' answer to the question, "How many days in the past week did you use snuff or chewing tobacco?" Players who answered that they used one or more days were considered to be current users. In the 2001 and 2002 surveys, players were also asked "In the past 7 days, have you used any spit tobacco, even just one dip or chew?" Using responses to this question gives slightly different point prevalence rates. In other publications of these data, these rates have been cited. However, in order to ensure continuity of the data over all six years we have chosen to report data from the first question here. 
Table 1 Seven day point prevalence of self reported smokeless tobacco use

\begin{tabular}{lllllll}
\hline & 1998 & 1999 & 2000 & 2001 & 2002 & 2003 \\
\hline Minor & $(n=1800)$ & $(n=1356)$ & $(n=1403)$ & $(n=2442)$ & $(n=2395)$ & $(n=2193)$ \\
& $31.7 \%$ & $26.3 \%$ & $25.7 \%$ & $25.9 \%$ & $27.0 \%$ & $24.8 \%$ \\
Major & $(n=312)$ & $(n=348)$ & $(n=454)$ & $N A$ & $(n=258)$ & $(n=136)$ \\
& $35.9 \%$ & $34.5 \%$ & $32.6 \%$ & - & $30.2 \%$ & $36.0 \%$ \\
\hline \multirow{7}{*}{ NA, not available. } & & & & & \\
\hline
\end{tabular}

off season. Fewer than half of both minor and major league players reported that they quit $(30-40 \%)$ or reduced $(35-$ $40 \%$ ) consumption when not playing baseball. Players reporting the same or greater use in the off-season also reported using ST on more days of the week $(M=5.81$, SD $=2.07)$, than players who quit in the off season $(M=3.60$, $\mathrm{SD}=2.41)\left(t(326)=8.49, \mathrm{p}<0.001, \mathrm{~d}=0.97, \eta^{2}=\right.$ 0.18 ). This same pattern held among both minor and major league players and suggests a greater level of dependence or addiction among those who use ST year round.

In 2000 and 2001, ST users were asked to rate their level of agreement with items reflecting motives for using ST. In both years, most players described their use as something to do, a habit or ritual, and approximately $40 \%$ indicated that they were addicted or that ST helped them to relax, other potential indicators of addiction and related withdrawal effects. There was a strong correlation between endorsing addiction as a reason for use and the amount of tobacco a player used. Players describing themselves as addicted to ST reported that their tins lasted fewer days $(r=-0.52, \mathrm{p}<0.001)$ and reported using ST on a greater number of days during the past week $(r=0.50, \mathrm{p}<0.001)$ than ST users who did not. Few players reported ST use as a coping strategy (such as chewing to deal with anger or depression), or as a concentration or weight control aid, and only $6 \%$ believed that ST helped to improve their on field performance.

Starting in 2000, the survey included questions on readiness to quit, reasons for quitting, and supports used to quit. Table 4 shows the responses of regular ST users from 2000 through 2003, combining major and minor league players. Findings show substantial interest in quitting among players, with $40-50 \%$ of players making a quit attempt at least once in the previous year. A contemplation ladder ${ }^{14}$ was used to assess players on where they fell along a continuum of readiness to quit ST, using the stages of change model. ${ }^{15}$ The majority of players were at least considering quitting, and many were taking action to quit. Only $11-12 \%$ of players reported no interest in quitting in any of the four years. The most frequently checked reasons for wanting to quit were concerns about the health effects of use, especially cancer. Over a third of players also noted concerns about negative role modelling, and almost as many reported that their significant other did not like it.

The surveys also asked about the helpfulness of various aids to (and supports for) quitting ST. Support from partner or spouse was the most commonly used support and was also rated as the most helpful. Players often reported that a dental exam and advice from a dentist were important aids in quitting (table 4). Many players had access to a dentist through NSTEP. NSTEP also provided motivational presentations on cessation methods and aids, and self-help quitting guides and materials on risks of ST use. Over a quarter of players indicated advice from an athletic trainer. PBATS and NSTEP activities for trainers stressed effective brief counselling, pharmacotherapy, and specialist referral as treatment options for players from 1998 through 2003. Surprisingly few players reported using pharmacotherapy or specialist counselling. Table 5 shows the percentages of ST users who participated in varied NSTEP programmes each year.

\section{Use of other tobacco products}

While our primary focus was on smokeless tobacco, we included questions on other forms of tobacco use. Thirty day point prevalence of cigarette and cigar smoking was substantially lower than the seven day point prevalence rates of ST usage (table 6).

Minor and major league players reported similar prevalence of cigarette smoking, whereas major league players reported significantly higher prevalence of cigar smoking (collapsing across years, eliminating 2001 because only minor league data are available, $\chi^{2}(1, \mathrm{n}=8326)=134.8$, $\mathrm{p}<0.001)$. Prevalence of cigarette smoking showed a significant decrease over time among both minor $\left(\chi^{2}(5, \mathrm{n}=\right.$ $11554)=117.7, \mathrm{p}<0.001)$ and major league players, $\left(\chi^{2}(4\right.$, $\mathrm{n}=1507)=012.5, \mathrm{p}<0.025)$. Prevalence of cigar smoking also showed significant downward trend over time among both minor $\left(\chi^{2}(5, \mathrm{n}=11554)=138.6, \mathrm{p}<0.001\right)$ and major league players, $\chi^{2}(4, \mathrm{n}=1505)=20.5$, $\mathrm{p}<0.001)$.

Cigarette and cigar smoking rates across race/ethnicity showed patterns similar to that of ST use. There were so few native American and Asian players that comparisons within these groups were not meaningful. Among white, Latino, and African American players, cigarette smoking is more prevalent among white players than among Latino and African American players. White players report about twice the prevalence of cigarette smoking (ranging from 5.5-11.0\% across the six annual surveys, major and minor league combined) as African American players (3.8-5.4\%), and about four times the prevalence reported by Latino players (1.7-3.0\%). African American players are most likely to report smoking cigars (3.5-14.7\%), followed by white (3.3-9.1\%) and then Latino players (2.1-6.8\%).

Table 2 Frequency of smokeless tobacco (ST) use

\begin{tabular}{llllllll}
\hline & & $\mathbf{1 9 9 8}$ & $\mathbf{1 9 9 9}$ & $\mathbf{2 0 0 0}$ & $\mathbf{2 0 0 1}$ & $\mathbf{2 0 0 2}$ & $\mathbf{2 0 0 3}$ \\
\hline Percentage of players reporting daily & Minor & $15 \%$ & $11 \%$ & $12 \%$ & $12 \%$ & $10 \%$ & $12 \%$ \\
ST use & Major & $21 \%$ & $19 \%$ & $17 \%$ & NA & $12 \%$ & $16 \%$ \\
Mean days used ST last week* & Minor & 5.3 & 5.0 & 5.0 & 4.9 & 4.7 & 5.1 \\
& Major & 5.7 & 5.2 & 5.4 & NA & 4.8 & 4.7 \\
Average number of days tin/pouch & Minor & NA & 4.4 & 4.7 & 4.4 & 4.7 & 4.5 \\
lasts* & Major & NA & 4.0 & 4.6 & NA & 4.1 & 4.4 \\
\hline
\end{tabular}

*Among those reporting use in past seven days. 
Table 3 ST use (seven day self reported point prevalence) by racial/ethnic group

\begin{tabular}{llllll}
\hline \multicolumn{5}{c}{ ST use (\% (n)) } \\
\cline { 2 - 5 } & 1999 & 2000 & 2001 & 2002 & 2003 \\
\hline $\begin{array}{l}\text { White } \\
\text { Latino }\end{array}$ & $36.2(369)$ & $34.3(386)$ & $33.0(479)$ & $38.0(569)$ & $30.1(458)$ \\
$\begin{array}{l}\text { African } \\
\text { American }\end{array}$ & $10.8(28)$ & $14.5(55)$ & $13.3(69)$ & $16.4(72)$ & $13.9(75)$ \\
Native & $20.3(27)$ & $14.3(20)$ & $17.1(32)$ & $21.4(34)$ & $19.0(28)$ \\
American & $47.1(8)$ & $47.1(8)$ & $36.0(9)$ & $52.4(11)$ & $62.5(10)$ \\
Asian & $0.0(0)$ & $14.3(2)$ & $28.6(6)$ & $26.9(7)$ & $30.3(10)$ \\
$\begin{array}{l}\text { Other } \\
\text { Response rate* }\end{array}$ & $17.0(45)$ & $22.0(9)$ & $25.7(9)$ & $10.7(3)$ & $31.3(10)$ \\
\hline $\begin{array}{l}\text { There were no appreciable differences in ST use by race between minor and major league players, therefore this } \\
\text { table collapses across league. "Other" represents respondents who classified their race in the category labelled }\end{array}$ \\
"other". \\
*Proportion of all respondents who reported race/ethnicity.
\end{tabular}

\section{DISCUSSION}

Annual surveys of professional baseball players were conducted from 1998 to 2003 to assess the prevalence of ST use over time. Major league players reported high prevalence of ST use compared to the general population of young adult males, with $30-36 \%$ of players reporting regular use across the six years of the survey, and no significant change in prevalence over time. A national survey of males ages 18-25 years in 1998 found that $10.5 \%$ had used ST in the past month, and by 2002 (the most recent year that nationwide prevalence rates are available) that percentage had dropped to $9.4 \% .{ }^{16}{ }^{17}$ Minor league players also reported a higher prevalence of ST use than the general population of young males, but a lower prevalence than among major league players, and a significant decline in use from 1998-2003, with the greatest drop (and a significant one) between 1998 and 1999 and rates of $24-27 \%$ thereafter. Minor league players were subject to stronger policy and environmental influences

Table 4 Readiness to quit, quit attempts, reasons for quitting, and quitting aids used among major and minor league players (percentage of regular ST users)

$200020012002 \quad 2003$

\begin{tabular}{|c|c|c|c|c|}
\hline \multicolumn{5}{|l|}{ Readiness to quit } \\
\hline No thought of quitting & 11.1 & 11.2 & 11.0 & 12.3 \\
\hline Think I need to consider quitting & 12.0 & 13.9 & 16.9 & 18.3 \\
\hline Think I should quit, but not quite ready & 36.7 & 29.0 & 25.4 & 26.6 \\
\hline $\begin{array}{l}\text { Starting to think about how to } \\
\text { change use }\end{array}$ & 27.2 & 30.7 & 31.9 & 32.5 \\
\hline \multicolumn{5}{|l|}{ Quit attempts } \\
\hline $\begin{array}{l}\text { Made one or more serious quit } \\
\text { attempts in past year }\end{array}$ & 51.2 & 51.4 & 41.9 & 40.1 \\
\hline \multicolumn{5}{|l|}{ Reasons for quitting } \\
\hline Chewing is hazardous to my health & 76.4 & 71.2 & 74.0 & - \\
\hline Don't want to get cancer & 64.4 & 58.3 & 61.4 & - \\
\hline It portrays a negative image to kids & 35.4 & 24.3 & 33.7 & - \\
\hline My significant other doesn't like it & 32.1 & 25.7 & 31.1 & - \\
\hline $\begin{array}{l}\text { It has unpleasant effects on my } \\
\text { appearance }\end{array}$ & 28.9 & 25.1 & 28.2 & \\
\hline Bad role model for my own kids & 28.1 & 16.5 & 26.6 & - \\
\hline The cost & 22.6 & 23.4 & 21.5 & - \\
\hline \multicolumn{5}{|l|}{ Quitting aids used } \\
\hline Support from spouse & 36.5 & 38.9 & 49.8 & - \\
\hline Advice from dentist & 29.3 & 26.8 & 34.1 & - \\
\hline Dental exam & 25.5 & 27.2 & 33.0 & - \\
\hline Support from other players & 23.5 & 25.2 & 30.2 & - \\
\hline Advice from trainer & 22.1 & 22.7 & 24.9 & - \\
\hline Nicotine gum & 11.3 & 10.9 & 16.9 & - \\
\hline Advice from specialist & 8.7 & 14.1 & 9.9 & - \\
\hline Nicotine patch & 8.1 & 8.7 & 8.9 & - \\
\hline Other nicotine replacement & 4.7 & 5.7 & 2.9 & - \\
\hline Bupropion (Zyban) & 3.2 & 5.2 & 2.9 & - \\
\hline
\end{tabular}

throughout the years of the surveys as there has been a ban on use of ST while in uniform and players can be fined for infractions. This policy has been well publicised but enforcement varies widely as it falls upon the umpire to cite the player. In addition, players coming from college teams would have been subject to the NCAA ban on ST use implemented in 1994. These findings suggest that sustained multi-level policy and programmatic efforts influenced ST use by minor leaguers. While the majority of players report using ST before becoming professional, $36 \%$ of minor league players report taking it up after they became pro.

In contrast to ST usage, baseball players report a substantially lower prevalence of cigarette smoking than the general population, both among major (14.4\% in 1998 to $10.3 \%$ in 2003 ) and minor ( $13.1 \%$ in 1998 to $4.2 \%$ in 2003$)$ league players. Nationwide rates of smoking cigarettes in the past month for males ages $18-24$ years were $31.3 \%$ in 1998 and $32.4 \%$ in $2002 .{ }^{16}{ }^{17}$ Cigar smoking rates were similar to rates among the general population. ${ }^{18}$ Lower prevalence of cigarette smoking among baseball players may be due to the increasing perception of smoking as an unhealthy habit, especially for athletes. It would seem that in the baseball culture, ST, although it has much potential for causing negative health effects, is seen as a more acceptable form of tobacco to use.

Given low and variable response rates and the possibility of self selection and self report biases, the results from these surveys may over- or underestimate actual ST prevalence among the professional baseball players surveyed. However, findings suggest substantial current prevalence at rates that are consistently to be higher than those in the general population of young males. Cigarette and cigar usage rates appear lower than typical among young males, indicating that ST remains the tobacco of choice for professional baseball players. This has been the trend dating back to heavy free sampling and player targeted marketing in the 1970 s by the US Tobacco Company. ${ }^{19}$

While ST use is in many ways tied to the game of baseball, the majority of players continue to use year round, consistent with the fact that many perceived themselves to be addicted and reported other signs of addiction. A majority of the players who used ST showed some interest in quitting. Many cited health concerns, effects on appearance, and the negative impact of their ST use on spouses and children as major reasons for wanting to quit. These findings show the need for continued intervention efforts.

The most frequent reasons players gave for using ST were that it gives them something to do, is a habit or ritual, and that they are addicted. As documented previously, very few players reported that they thought their playing was improved by ST use. $^{19}$ This is consistent with previous research showing no relation between ST use and motor performance tasks. ${ }^{20}$ 
Table 5 Reported exposure to components of NSTEP (percentage of regular ST users)

\begin{tabular}{lcccccc}
\hline & 1998 & 1999 & $\mathbf{2 0 0 0}$ & $\mathbf{2 0 0 1}$ & $\mathbf{2 0 0 2}$ & $\mathbf{2 0 0 3}$ \\
\hline Presentation to players & $*$ & 38.6 & 33.7 & 33.4 & $*$ & $*$ \\
Cessation counselling & 14.9 & $*$ & 18.3 & 21.8 & 32.6 & 29.3 \\
Oral exams & 35.0 & 34.2 & 41.3 & 38.3 & 47.2 & 35.7 \\
Materials on risk & $*$ & 26.2 & 31.9 & 49.1 & $25.3 \dagger$ & $29.4 \dagger$ \\
Materials on quitting & 41.7 & 20.8 & 29.5 & 35.1 & $25.3 \dagger$ & $29.4 \dagger$ \\
Pictures of oral health problems & 21.7 & 55.6 & $*$ & $*$ & $*$ & $*$ \\
\hline
\end{tabular}

*Programme was not available.

tln 2002 participants were asked just one question on whether they had received materials on risk or quitting; this number represents the percentage that responded affirmatively to this question.

Players who use ST reported that their dentists seldom pointed out lesions or counselled them on ST cessation. Previous research has shown interventions by dentists and dental hygienists in the context of oral health care to be effective in motivating ST cessation. ${ }^{71} 22$ Since a high percentage of daily users of snuff have observable oral lesions, pointing out these lesions provides a "teachable moment" that can result in significant cessation of ST use. ${ }^{22} 23$ A policy of required oral exams and training of team affiliated dentists at the local level could provide an effective channel of intervention.

It is encouraging that most players report interest in quitting and that $50 \%$ of players reported one or more serious quit attempts in the past year. But most players at spring training were not ready to quit (in action stages); this is a high pressure time for them as they strive to win promotion to a higher rated team. This and the high rates of year round ST use suggests the need for ST education and treatment efforts that span the entire season and whole year. Recent developments using computer technology may further this goal and they include a web based ST cessation programme sponsored by the National Cancer Institute (chewfree.com), a PBATS sponsored internet programme for athletic trainers at all levels (with CME credit), and computer based games for ST cessation (for example, Chewers Choice $^{24}$ ).

This study has several limitations. First, it is a sequential cohort study, thus we have no longitudinal data to enable us to look at changes in individual player's behaviour. Our sample each year is made up of volunteering subjects from the teams and represents fewer than $50 \%$ of the players in any year. We could not evaluate the representativeness of our samples; it is possible that tobacco users are either under- or over-represented in the study as participation was voluntary. Also, all data are self reported, and there was no independent verification of ST use.

On the positive side, the repeated assessments under the same conditions at the same time of the year provide a solid basis for estimation of trends and co-variation, and this is the most systematic long term survey of professional baseball players ever conducted. While trends across time are less likely to be affected by low response rate, NSTEP programmes may have contributed to under reporting of use. While baseball players continue to have high levels of ST use, there is some evidence that use among minor league players

\section{What this paper adds}

This is the largest dataset of baseball players ever collected and the use of smokeless and other tobacco products was surveyed over a six year period. The paper provides new data on prevalence, changes in attitudes, and prevalence and associated variables that provide new information on a group of users whose use can influence young people to use snuff or chew by viewing these role models through the media.

dropped after players were exposed to an educational programme about the dangers of using ST. Given the prominent place that baseball has in US culture, and the potential influence that players can have on young people who look to them as sports idols, it is important to continue the effort to help players quit, and change the policy and environmental influences that support ST use and association with the culture of baseball. While major league baseball, NSTEP, and the Professional Baseball Athletic Trainers Society have taken major steps to educate players and trainers, and provide oral screening and cessation assistance over the past six years, there remains more work to do. This change in the culture of ST use will be slow but it could be enhanced by enforcing restrictions on players use on the field, and extending these restrictions to major league players as well as minor league players. Such restrictions have so far been resisted by the players union, which sees this as a "player's right" to use tobacco. It is likely that with increasing environmental restrictions on tobacco use we may see, one day, the baseball field as a "no tobacco zone".

\section{ACKNOWLEDGEMENTS}

We would like to thank the Professional Baseball Athletic Trainers Society (PBATS) for their support in making this research possible. Thanks especially to the PBATS tobacco committee members, Richie Bancells, Ned Bergert, Jeff Cooper, and to the presidents of PBATS during the years these surveys were conducted: Russ Miller, Jamie Reed. Thanks also to Major League Baseball, the National Spit Tobacco Education Program, and Romano and Associates for supporting our work on this project.

This research was funded by a grant from the Robert Wood Johnson Foundation No. 044095.

Table 6 Smoking in past 30 days: major and minor league players 1998 to 2003

\begin{tabular}{llllllll}
\hline & $\mathbf{1 9 9 8}$ & $\mathbf{1 9 9 9}$ & $\mathbf{2 0 0 0}$ & $\mathbf{2 0 0 1}$ & $\mathbf{2 0 0 2}$ & $\mathbf{2 0 0 3}$ \\
\hline Minor & & $\mathrm{n}=1800$ & $\mathrm{n}=1356$ & $\mathrm{n}=1403$ & $\mathrm{n}=2442$ & $\mathrm{n}=2395$ & $\mathrm{n}=2193$ \\
& Smoke cigarettes & $13.1 \%$ & $7.5 \%$ & $7.8 \%$ & $7.8 \%$ & $6.3 \%$ & $4.2 \%$ \\
\multirow{4}{*}{ Major } & Smoke cigars & $10.1 \%$ & $6.5 \%$ & $5.3 \%$ & $4.8 \%$ & $3.3 \%$ & $2.6 \%$ \\
& & $\mathrm{n}=312$ & $\mathrm{n}=348$ & $\mathrm{n}=454$ & $\mathrm{NA}$ & $\mathrm{n}=258$ & $\mathrm{n}=136$ \\
& Smoke cigarettes & $14.4 \%$ & $8.1 \%$ & $7.9 \%$ & & $7.5 \%$ & $10.3 \%$ \\
& Smoke cigars & $18.9 \%$ & $18.4 \%$ & $13.0 \%$ & & $10.1 \%$ & $6.6 \%$ \\
\hline
\end{tabular}




\section{Authors' affiliations}

H H Severson, K Klein, E Lichtensein, N Kaufman, Oregon Research Institute, Eugene, Oregon, USA

C T Orleans, Robert Wood Johnson Foundation, Princeton, New Jersey, USA

\section{REFERENCES}

1 Greene JC, Walsh MM, Letendre MA. Prevalence of spit tobacco use across studies of professional baseball players. J California Dental Assoc 1998:26:358-64

2 Wisniewski JF, Bartolucci AA. Comparative patterns of smokeless tobacco usage among major league baseball personnel. J Oral Pathol Med 1989; 18:322-6.

3 Sinusas K, Coroso JG, Sopher MD, et al. Smokeless tobacco use and oral pathology in a professional baseball organization. J Family Pract 1992;34:713-18.

4 Jones RB. Use of smokeless tobacco in the 1986 World Series. N Engl J Med 1987:316:952.

5 Sussman S, Barovich M. Smokeless tobacco: less seen at 1988 World Series. Am J Public Health 1989;79:521-22.

6 Jones R. Use of smokeless tobacco in the World Series, 1986 through 1993 Am J Public Health 1995;85:117.

7 Walsh MM, Green JC, Ellison JA, et al. A dental-based, athletic trainermediated spit tobacco cessation program for professional baseball players. J California Dental Assoc 1998;26:365-76.

8 Walsh MM, Hilton JF, Ernster VL, et al. Prevalence, patterns and correlates of spit tobacco use in a college athlete population. Addictive Behaviors 1994;19:411-27.

9 Levenson-Gingiss P, Gottlieb NH. A comparison of smokeless tobacco and smoking practices of university varsity and intramural baseball players. Addictive Behaviors 1991;16:335-40.

10 Wisniewski JF, Mohl GR, Shedroff DM. Smokeless tobacco use by high school baseball players. Health Education 1990;21:10-15.
11 Ernster VL, Grady DG, Stillman L, et al. Smokeless tobacco in professional baseball: Patterns of players' use. Smoking and Tobacco Control Monograph No. 21992.

12 Ernster VL, Grady DG, Greene JC, et al. Smokeless tobacco use and health effects among baseball players. JAMA 1990;264:218-24.

13 Ban on smokeless tobacco expanded to single " $A$ " ball. PBATS Newsletter 1992;5:7.

14 National College Athletic Association. Rule 11.1.7. Use of tobacco products adopted 11 January, 1994.

15 Connolly GN, Garagiola J. Commentary: It's time Major League Baseball made tobacco history. J Am Dental Assoc 1995; 126:1121-4.

16 Centers for Disease Control and Prevention. Cigarette smoking among adults United States, 1998. MMWR Morb Mortal Wkly Rep 2000:49:881-4.

17 Centers for Disease Control and Prevention. Cigarette smoking among adultsUnited States, 2002. MMWR Morb Mortal Wkly Rep 2004;53:427-31.

18 Substance Abuse and Mental Health Services Administration. Summary of findings from the 2000 National Household Survey on Drug Abuse (National Household Survey on Drug Abuse Series: H-13. Rockville, Maryland: DHHS (Publication No, SMA 01-3549), 2000.

19 Connolly GN, Orleans CT, Kogan M. Use of smokeless tobacco in Major League Baseball. N Engl J Med 1988;318:1281-5.

20 Edwards SW, Glover ED, Schroeder KL. The effects of smokeless tobacco on heart rate and neuromuscular reactivity in athletes and nonathletes. The Physician and Sports Medicine 1987; 15(7):141-6.

21 Severson HH, Andrews JA, Lichtenstein E, et al. A self-help cessation program for smokeless tobacco users: comparison of two interventions. Nicotine Tob Res 2000;2:363-70.

22 Gordon JS, Severson HH. Tobacco cessation through dental office settings. J Dental Educ 2001;65:354-63.

23 Little SJ, Stevens V, LaChance P, et al. Smokeless tobacco habits and oral mucosal lesions in dental patients. J Public Health Dentistry 1992:52:269-76.

24 Severson HH, Christiansen S, Jacobs T. Chewer's Choice: Interactive CD-ROM software for smokeless tobacco cessation [computer software]. Deschutes Research Institute and InterVision. Eugene, Oregon: Applied Behavior Science Press, copyright, 2002.

\section{The Lighter Side}

\section{SPEED BUMP}

\section{Dave Coverly}

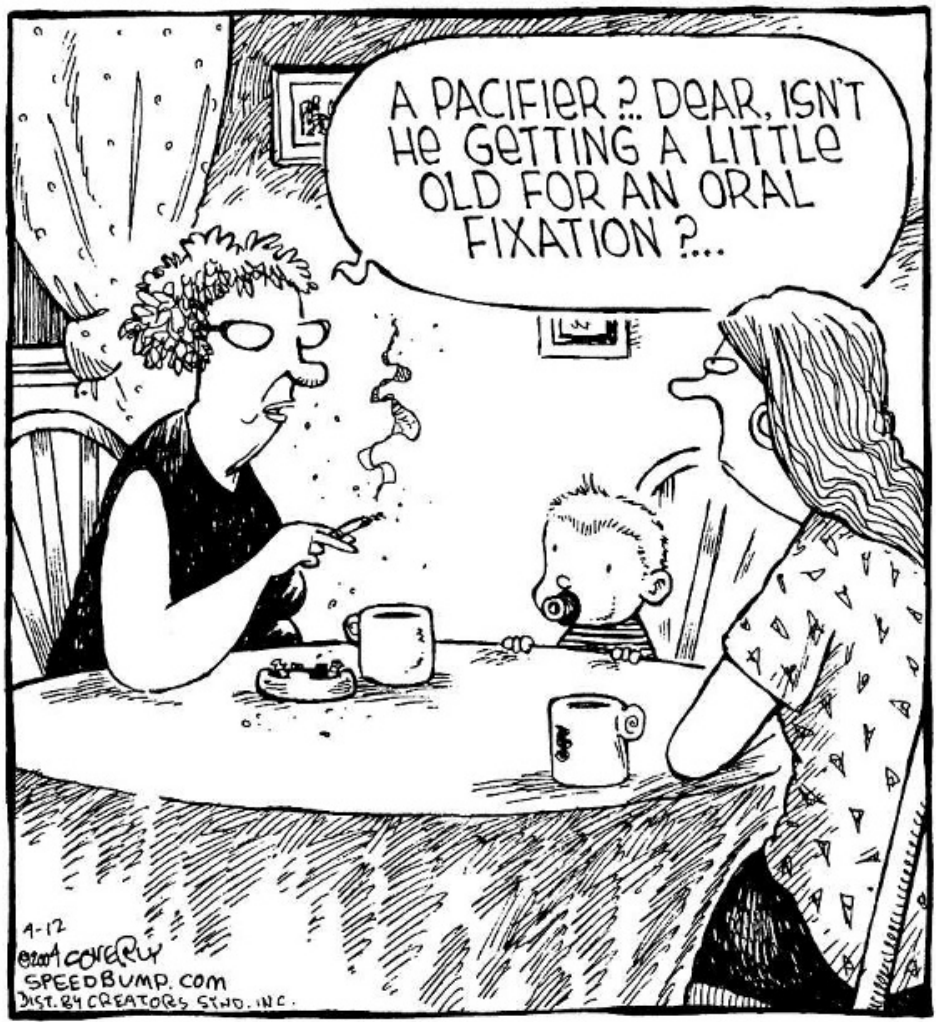

(C) By Permission of Dave Coverly and Creators Syndicate, Inc 